\title{
A Version of Jung's Synchronicity in the Event of Correlation of Mental Processes in the Past and the Future: Possible Role of Quantum Entanglement in Quantum Vacuum
}

\author{
Igor V. Limar
}

\begin{abstract}
This paper deals with the version of Jung's synchronicity in which correlation between mental processes of two different persons takes place not just in the case when at a certain moment of time the subjects are located at a distance from each other, but also in the case when both persons are alternately (and sequentially, one after the other) located in the same point of space. In this case, a certain period of time lapses between manifestation of mental process in one person and manifestation of mental process in the other person. Transmission of information from one person to the other via classical communication channel is ruled out. The author proposes a hypothesis, whereby such manifestation of synchronicity may become possible thanks to existence of quantum entanglement between the past and the future within the light cone. This hypothesis is based on the latest perception of the nature of quantum vacuum.
\end{abstract}

Key Words: light cone, Bogolyubov coefficients, Rindler wedges, synchronicity, quantum entanglement, quantum vacuum

\begin{abstract}
Problem formulation and related studies

The synchronicity phenomenon described by Carl Gustav Jung remains of contemporary interest, and the study of this phenomenon continues until present time. One of the main hypotheses involves assumption of existence of quantum entanglement (quantum nonlocality, quantum coherence) of the objects of microworld in one person with the similar objects of microworld in the brain of another person (Herbert, 1988; Carminati and Martin, 2008; Haas, 2010; Petrenko, 2010; Brizhik et al., 2011; Caramel and Stagnaro, 2011a; Caramel and Stagnaro, 2011b; Fach, 2011; Gernert, 2011; Haas, 2011; Levin, 2011; Martins, 2011; Schöter, 2011; Walach and Stillfried, 2011). The most important aspect of this problem is to determine how exactly
\end{abstract}

Corresponding author: Igor V. Limar

Address: Institute of Innovative and Postgraduate Education (IIPE). Department of computer science and informational technologies, Dvoryanskaya str., 2, Odessa, 65026, Ukraine.

Phone: +38048725 3687

Fax: -

$\bowtie$ iv.limar@onu.edu.ua (using what particular physical mechanism) the quantum entanglement may appear between molecules of one person's brain and biological molecules of another person. The author of (Limar, 2011) has suggested that quantum entanglement between biological molecules of two different persons may appear at the very moment when biological cells are divided during meiosis. However, the latest studies of the nature of quantum vacuum (Olson and Ralph, 2011) which were recently published propose to consider a possibility of correlation of mental processes in one person with mental processes in another person because of quantum entanglement between the past and the future within the light cone.

\section{Study goal and hypothesis}

As follows from the paper (Olson and Ralph, 2011), quantum entanglement may manifest itself not only at the same moment of time, existing between two different objects of microworld located at a certain distance from each other. The above paper proves that quantum entanglement between the past and the future may exist in one point of space, 
taking into account modes of quantum vacuum in which energy continuously fluctuates and electron-positron pairs are continuously created and annihilated. It is assumed that this effect may exist due to the fact that Bogolubov coefficients used in field quantization are formally absolutely similar for two different cases. The first case involves existence of quantum entanglement in quantum vacuum between the left and right Rindler wedges. The other case represents analysis of the state of quantum vacuum in the very same point of space in past and future moments within the light cone. As follows from the paper (Olson and Ralph, 2011), a detector located in a certain point of space may record the change of parameters we measure. In this case, the change of these parameters is caused by the event (fluctuation of quantum vacuum) taking place at a certain moment of time in the past, whereas the measuring is considered a moment in the future within the light cone. In other words, a certain period of time is lapsing between the moment when fluctuation of quantum vacuum takes place in a given point of space and the moment when detector measures parameters. Connection between the change of parameter measured in a given point of space and fluctuation of quantum vacuum in the same point of space in the past is caused by quantum entanglement. Therefore, we cannot rule out the fact that certain molecules of human brain may respond to the state of quantum vacuum in certain points of space. In turn, the state of quantum vacuum in these points of space may change through interaction with quantum vacuum of brain molecules in another person who previously was in the same points of space. To be sure, this phenomenon cannot explain all cases of synchronicity but only those between which a certain period of time has lapsed. In addition, if a short time has lapsed between the stay of one person in a certain point of space and the stay of another person in the same point of space, one may get an illusion of manifestation of synchronicity at the same moment of time. However, one may also assume that response of human brain to the state of quantum vacuum may be delayed. In other words, the stay of a person in a certain point of space may become a triggering mechanism (impulse) launching a chain of molecular processes in the human brain. But the synchronicity phenomenon per se will manifest itself only some time later (perhaps over quite lengthy period) after the quantum vacuum will have its effect on the brain. In any case, in the current phase of scientific development we have no answer to the question of whether such quantum entanglement may transform into an ordinary quantum entanglement between molecules of one person's brain and biological molecules of another person's brain. It is also worth noting that if such instances of biological molecules in different people interacting through quantum vacuum do take place, they probably have to be selective. It means that apparently, molecules of the human brain do not always have to respond to the state of quantum vacuum caused by the previous stay of other persons in a given point of space. Such selectiveness should apparently be caused by individual differences in brain structures which (the differences), in turn, are caused by genes. At the same time, unlike in the paper (Limar, 2011), the situation this paper deals with does not allow to determine in this phase of study the localization of brain structures and structures of nerve cells which may develop synchronicity. While the paper (Limar, 2011) unambiguously determines that these structures are represented by molecules, which become biologically active during mitosis, here we cannot determine such molecules yet.

Finally, we may add that other authors have previously studied the correlation of phenomena in quantum vacuum with manifestation of mental processes (Laughlin, 1996; Pratt, 2003). Perhaps we should also mention the so-called Boltzman brain paradox, which has to do with the quantum vacuum and consciousness (Albrecht and Sorbo, 2004; Linde, 2007; Bousso et al., 2008; Page, 2008; Simone et al., 2010). The phenomenon described by Russian scientist Gariaev may be of interest as well. It involves the so-called 'phantom effect' - a phenomenon whereby the space register a 'trace' of biological molecule (DNA) sometime after the biological molecule was moved from that point of space (Gariaev et al., 2011a; Gariaev et al., 2011b; Gariaev et al., 2011c; Gariaev and Pitkanen, 2011). It is quite possible that the 'trace' of molecule may be caused by the 'memory' of quantum vacuum. French scientist Montagnier has arrived at similar conclusions (Montagnier et al., 2009a; Montagnier et al., 2009b). And although in Montagnier's experiments the trace of DNA 
molecule was 'memorized' in the water medium, theoretical substantiation of this phenomenon is based on the properties of quantum vacuum (Arani et al., 1995), particularly applicable to biological molecules Giudice et al., 2005; Giudice et al., 2010).

\section{Conclusions and prospects of this study} As of today, the situation dealt with here presents more questions than answers. Of course, we cannot be sure that the proposed hypothesis will be proved by experiments. In turn, experimental verification involves substantial difficulties, because we are talking about complex biological molecules, while specific molecules themselves (from among the huge number of possible candidates) remain unknown. At the same time, we have to allow for possible existence of the mechanism described herein, even taking into account possible subsequent experimental disproval of this assumption. Despite the existence of several opinions concerning quantum entanglement in time (Godunov and McGuire, 2001; Godunov et al., 2001; McGuire et al., 2001; Merabet et al., 2001; McGuire et al., 2003; Soubusta et al., 2005), the paper (Olson and Ralph, 2011) deserves special attention.
References

Albrecht A and Sorbo L. Can the universe afford inflation? Physical Review D 2004; 70: 063528.

Arani R, Bono I, Giudice ED and Preparata G. QED coherence and the thermodynamics of water. International Journal of Modern Physics B 1995; 9: 1813-1841.

Bousso R, Freivogel B and Yang I. Boltzmann babies in the proper time measure. Physical Review D 2008; 77: 103514.

Brizhik LS, Giudice ED, Tedeschi A and Voeikov VL. The role of water in the information exchange between the components of an ecosystem. Ecological Modelling 2011; 222: 2869-2877.

Caramel S and Stagnaro S. Quantum biophysical semeiotics and mitgenome's fractal dimension. Journal of Quantum Biophysical Semeiotics 2011a; 1: 1-27.

Caramel S and Stagnaro S. Quantum chaotic aspects of biophysical semeiotics. Journal of Quantum Biophysical Semeiotics 2011b; 1: 28-70.

Carminati GG and Martin F. Quantum mechanics and psyche. Physics of Particles and Nuclei 2008; 39: 560-577.

Fach W. Phenomenological aspects of complementarity and entanglement in exceptional human experiences. Axiomathes 2011; 21: 233-247.

Gariaev PP, Friedman MJ and Leonova-Gariaeva EA. Principles of linguistic-wave genetics. DNA Decipher Journal 2011a; 1: 011-024.

Gariaev PP, Kokaya AA, Leonova-Gariaeva EA, Muldashev ER, Smelov MV, Tertishny GG and Ustinova NV. Exploration of wavegenetics and wave immunity. DNA Decipher Journal 2011b; 1: 191-217.

Gariaev PP, Marcer PJ, Leonova-Gariaeva KA, Kaempf U and Artjukh VD. DNA as basis for quantum biocomputer. DNA Decipher Journal 2011c; 1: 025-046.

Gariaev PP and Pitkänen M. Model for the findings about hologram generating properties of DNA. DNA Decipher Journal 2011; 1: 047-072.

Gernert D. Distance and similarity measures in generalised quantum theory. Axiomathes 2011; 21:303-313.

Giudice ED, Ninno A, Fleischmann M, Mengoli G, Milani M, Talpo G and Vitiello G. Coherent quantum electrodynamics in living matter. Electromagnetic Biology and Medicine 2005; 24: 199-210.

Giudice ED, Spinetti PR and Tedeschi A. Water dynamics at the root of metamorphosis in living organisms. Water 2010; 2: 566-586.

Godunov AL and McGuire JH. Independent time approximation for dynamically interacting multi-electron systems. Journal of Physics B 2001; 34: L223-L229.

Godunov AL, McGuire JH, Ivanov PB, Shipakov VA, Merabet H, Bruch $\mathrm{R}$, Hanni $\mathrm{J}$ and Shakov KK. Spatial and temporal correlation in dynamic, multi-electron quantum systems. Journal of Physics B 2001; 34: 5055-5069.

Haas AS. On a physical scientific approach to transpersonal psychology. International Journal of Transpersonal Studies 2011; 30: 69-81.

Haas AS. The interpretation of telepathy-like effects: a novel electromagnetic and synchronistic version of the psychoanalytic model. NeuroQuantology 2011; 9: 22-35.
Herbert N. How Bell proved reality cannot be local. Psychological Perspectives 1988; 19: 313-319.

Laughlin CD. Archetypes, neurognosis and the quantum sea. Journal of Scientific Exploration 1996; 10: 375-400.

Levin T. Holographic trans-disciplinary framework of consciousness: an integrative perspective. Journal of Consciousness Exploration \& Research 2011; 2:1385-1416.

Linde A. Sinks in the landscape, Boltzmann brains and the cosmological constant problem. Journal of Cosmology and Astroparticle Physics 2007; 2007/01: 022.

Limar IV. Carl G. Jung's synchronicity and quantum entanglement: Schrödinger's cat 'wanders' between chromosomes. NeuroQuantology 2011; 9: 313-321.

Martins PN. Science and the art of healing: a contribution to the history of life science. World Futures 2011; 67: 500-509.

McGuire JH, Godunov AL, Shakov KK, Shipakov VA, Merabet H, Bruch $\mathrm{R}$ and Hanni J. Time ordering in multi-electron dynamics. Journal of Physics B 2003; 36: 209-216.

McGuire JH, Godunov AL, Tolmanov SG and Shakov KK, Dörner R, Schmidt-Böcking $\mathrm{H}$ and Dreizler RM. Time correlation in twoelectron transitions produced in fast collisions of atoms with matter and light. Physical Review A 2001; 63: 052706.

Merabet H, Bruch R, Hanni J, Godunov AL and McGuire JH. Simultaneous ionization-excitation of helium to $\mathrm{He}+(2 \mathrm{p})$ magnetic sublevels by proton impact. Physical Review A 2001; 65: 010703 .

Montagnier L, Aissa $\mathrm{J}$, Ferris S, Montagnier $\mathrm{J}$ and Lavallee C. Electromagnetic signals are produced by aqueous nanostructures derived from bacterial DNA sequences. Interdisciplinary Sciences: Computational Life Sciences 2009a; 1: 81-90.

Montagnier L, Aissa J, Lavallee C, Mbamy M, Varon $\mathrm{J}$ and Chenal $\mathrm{H}$. Electromagnetic detection of HIV DNA in the blood of AIDS patients treated by antiretroviral therapy. Interdisciplinary Sciences: Computational Life Sciences 2009b; 1: 245-253.

Olson SJ and Ralph TC. Entanglement between the future and the past in the quantum vacuum. Physical Review Letters 2011; 106: 110404.

Page DN. Return of the Boltzmann brains. Physical Review D 2008; 78 : 063536.

Petrenko VF. Toward the problem of the psychology of the consciousness. Voprosy Filosofii 2010; 11: 57-74. (in Russian)

Pratt D. Consciousness, causality, and quantum physics. NeuroQuantology 2003; 1: 58-67.

Schöter A. The Yijing: metaphysics and physics. Journal of Chinese Philosophy 2011; 38: 412-426.

Simone AD, Guth AH, Linde A, Noorbala M, Salem MP and Vilenkin A. Boltzmann brains and the scale-factor cutoff measure of the multiverse. Physical Review D 2010; 82: 063520.

Soubusta J, Perina J, Haderka O, Hendrych M and Dusek M. Experimental tests of energy and time entanglement. Acta Physica Hungarica B 2005; 23: 143-150.

Walach $\mathrm{H}$ and Stillfried N. Generalised quantum theory-basic idea and general intuition: a background story and overview. Axiomathes 2011; 21: 185-209. 